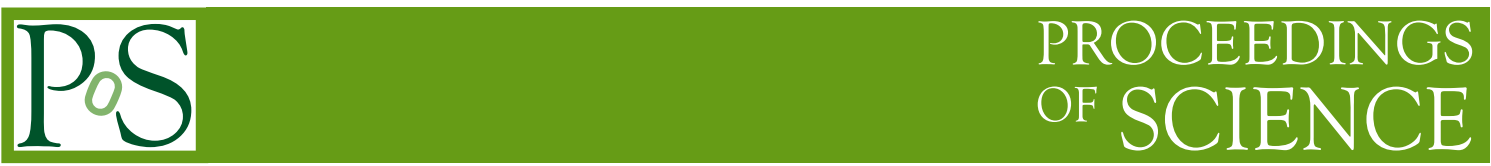

\title{
Observers diffeomorphism-invariant description of a general relativistic system
}

\section{Jędrzej Swieżewski*}

Faculty of Physics, University of Warsaw

E-mail: swiezew@fuw.edu.pl

I will present a construction of observables following naturally from an introduction of an observer into a relativistic theory. The observables are invariant under a large class of spatial diffeomorphisms. I will discuss the description of the theory in terms of spatial-diffeomorphisminvariant degrees of freedom. The talk will be based on [1].

References

[1] P. Duch, W. Kaminski, J. Lewandowski and J. Swieżewski, Observables for General Relativity related to geometry, arXiv:1403.8062 [gr-qc].

Frontiers of Fundamental Physics 14 - FFP14,

15-18 July 2014

Aix Marseille University (AMU) Saint-Charles Campus, Marseille

${ }^{*}$ Speaker. 